\title{
THE RESEARCH OF ROUTE NAVIGATION BASED ON VISUAL NAVIGATION
}

\author{
Zhaobin Peng ${ }^{1}$, Lijuan Wang ${ }^{1}$, Yaru Zhang ${ }^{1}$, Yuanyuan ${ }^{1}$, Fangliang An ${ }^{1}$, \\ Rongjun Zhang , Yaoguang Wei \\ ${ }^{I}$ Department of Information and Electrical Engineering, China Agricultural University, \\ Beijing, P. R. China 100083 \\ * Corresponding author, Address: Department of Information and Electrical Engineering, \\ China Agricultural University, Beijing 100083, P. R. China, Tel: 13521591976, Email: \\ wygzz@yahoo.com.cn
}

Abstract: In order to solve the problems of Image pretreatment and the extraction of navigation line on agricultural machinery visual navigation, a route navigating system based on improved HOUGH transform is brought forward. First, extract the set of points from the original image based on the threshold segmentation and edge detection. Second, an improved HOUGH transform algorithm is used on detecting the route. This system is based on DM642 DSP and ARM9 Integrated Development Environment, which have achieved satisfactory experimental results.

Keyword: Visual navigation, threshold segmentation, edge extraction, improved HOUGH transform

\section{INTRODUCTION}

The visual navigation(Gan-Mor S et at., 2001) approach is one of the advanced navigation methods boomed up in recent years, which incorporates many advantages such as less noise, less harmful effects in comparison with non-visual sensors. In practical applications, drawing the leading path is all that's necessary, a robot can follow the path through visual navigation system all by itself (Pinto et at., 2000) (Toru et at., 2000) .

\footnotetext{
Please use the following format when citing this chapter:
}

Peng, Z., Wang, L., Zhang, Y., Yuanyuan, An, F., Zhang, R. and Wei, Y., 2009, in IFIP International Federation for Information Processing, Volume 294, Computer and Computing Technologies in Agriculture II, Volume 2, eds. D. Li, Z. Chunjiang, (Boston: Springer), pp. 1511-1517. 
Straight line detection is an important approach in image processing(Zhao Ying et at., 2006), in which Hough Transform is commonly adopted. The Hough transform (HT) (Zhang Wei et at., 2005), in essence, is a method for grouping pixels in certain connections and searching for a parameterization to establish an equation by using the corresponding points of these pixels in the parameter space. But since this algorithm can not meet the real-time requirement(Illingworth $\mathrm{J}$ et at., 1988), the paper have proposed a visual navigation system, which provides visual-guiding for robot based on path guiding lines and marked identification(Wilson et at., 2000). In order to ameliorate the system, the real-time algorithm and robust are fully taken into account in each step from image preprocessing through path recognition and tracking.

We dig a little deeper into this question, and come up with the solution based on multi-known points Hough transform, increasing the processing speed of the real-time system.

\section{IMAGE PRETREATMENT}

\subsection{Median filter}

Median filter method was originally developed by J.W.Jukey in 1971 to process signal. Median filter is a non-linear image smoothing method, which is used to remove the high-frequency noise such as the Salt and Pepper noise and can better keep the edges from being blurred. The image is equipped with a small window, which of size with odd number of pixels, within the window, replacing each of them with the median value acquired from the middle pixel of the sequence rearranged in order of their gray value.

\subsection{Edge detection ( Sobel operator)}

The edge detector inspect the neighborhood of each pixel, and quantify the gray rate, as well as the determination of the orientation. Most method employ the directive differentiation mask convolution.

Fig.1 shows the Sobel operator composed of two convolution nuclei, each pixel in the image is to be convoluted by these nuclei, one nucleus are sensitive to the vertical edge, the second nucleus, on the other hand, can detect the horizontal edge. The maximum value of the convolution results is output as the representative of this pixel. The effect of this algorithm is to get the contour of the image. 


\begin{tabular}{|c|c|c|}
\hline-1 & -2 & -1 \\
\hline 0 & 0 & 0 \\
\hline 1 & 2 & 1 \\
\hline
\end{tabular}

\begin{tabular}{|l|l|l|}
\hline-1 & 0 & 1 \\
\hline-2 & 0 & 2 \\
\hline-1 & 0 & 1 \\
\hline
\end{tabular}

Fig. 1: Template of Sobel

By using Sobel operator, the detected edge is clear. Sobel operator has always been chosen to detect the edge.

\subsection{Threshold segmentation}

After the edge detection, the threshold algorithm is done to the image, so the detail of the image is discernable.

\section{EXTRACTION OF NAVIGATION LINE}

The path recognition is the primary goal of making scientific researches on Agriculture autonomous walking robot visual navigation which usually represents as striated target. In the farmland environment, straight lines or lines slightly curved, the navigation line can be assumed to be straight. By processing the results of image analysis, navigation path can be isolated. Line detection is the important part in image analysis and computer vision. The most commonly used method is the least-squares and HOUGH transform. The least squares method is sensitive to the noise, and the classical HOUGH transform processing speed is very slow. A known point of HOUGH transform is faster, but is unable to the known point to carry on the accurate localization. For the above-mentioned problems, This article proposed based on multi-points HOUGH transform.

\subsection{The Classical Hough transform}

In 1962 Paul Hough first proposed the HOUGH transform, which realized one kind of mapping from the image space to the parameter space. The fundamental thought is duality between pixels and lines, namely the collinear point in image space corresponds to intersectant straight line in parameter space. Meanwhile, all straight line intersecting in a spot in parameter space corresponds to the collinear spot in the image space.

Since the straight line slope may be infinite, straight line represented by Polar coordinate equation is always used in the Hough transform, namely expressing the point of the straight line in the image space with the sine curve. The equation is as follows: 
$\rho=x * \cos \theta+y^{*} \sin \theta, \rho \in R \quad \theta \in[0,2 \pi]$
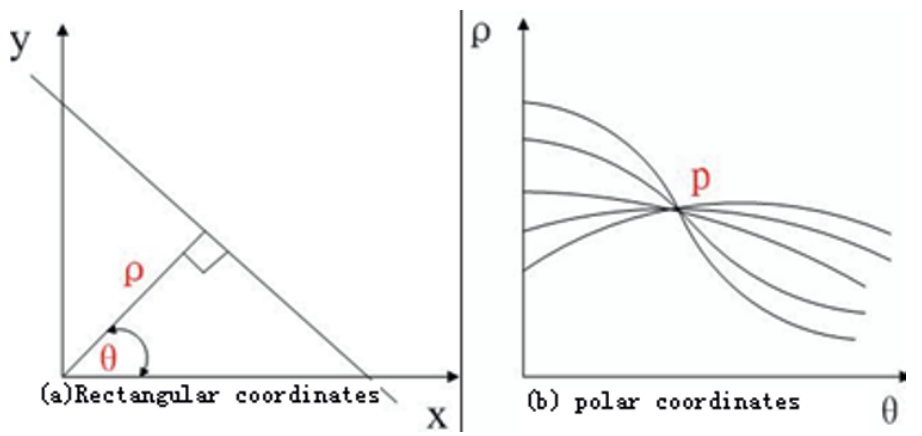

Fig.2: Change of Parameter between Rectangular coordinates and Polar coordinates

When all characteristic points in a straight line of a image have been carried out in this transformation, in the parameter space there will be many sine curves, all of which pass through $(\rho, \theta)$. So that the parameters on a straight line can be expressed by ${ }^{(\rho, \theta)}$ unit's coordinate, shown as picture b. The following two pictures are the results of a single image's HOUGH transform:

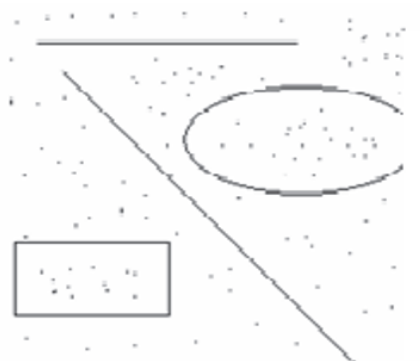

(a) Original image

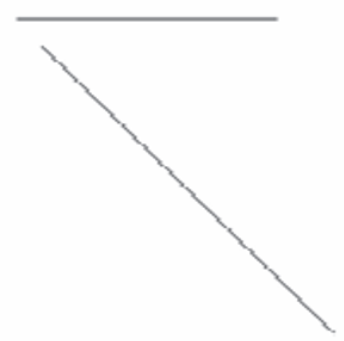

(b) Resulting image

Fig.3: Result of Classic Hough transform

The extraction of straight line is accurate by using Hough transform, but it has a very serious shortcoming: It can't detect specific curve, and it's difficult to find the local maximum value of accumulator array, low precision, the large storage space. In order to solve these problems, the people proposed Hough transform with a known point.

\subsection{The Hough transform with a known point}

The Hough transform with a known point is a kind of improvement algorithm to the classical Hough transform .Firstly, finding a point on the 
line which will be defined as $\rho_{0}$ (the known point). Define this known point's coordinate as $\left(x_{0}, y_{0}\right)$ and the slope of the straight line through $\rho_{0}$ as $\mathrm{m}$, then the relationship between the slope and coordinate can be expressed by the following equation:

$$
m_{i}=\left(y_{i}-y_{0}\right) /\left(x_{i}-x_{0}\right) .
$$

Map the slope value to a group of accumulators, making its corresponding accumulator's value to add 1 when obtains a slope each time. Each point on the same straight line has identical slope, so when there has straight line ingredient in the target area, its corresponding accumulator has the local maximum. The corresponding slope is taken as the straight line slope.

The Hough transform with a known point can greatly improve the computing speed, but the key issues is how to select the point correctly. It is random and the direction is uncertain in a real-time image. Taking into account the above issues, the method of Hough transform with multi-known points was adopted.

\subsection{Improved HOUGH transform algorithm}

From the above discussion we can see that the classical HOUGH transform and a known point HOUGH transform are not feasible to extract a straight line from real-time video clips. So the improved HOUGH transform algorithm based on multi-known points is adopted. Generally, the route may pass through the entire image. After several rounds of the image pretreatment, there are always bright spots on the 100th row for each image. After the pretreatment, very little noise is left. The following description tells the process of the whole experiment in detail.

\section{THE APPROACH OF THE RECOGNITION ALGORITHM}

The approach of the entire experiments are described as follows:

(1) Image pretreatment. Getting the binarization image with few noise

(2) Eliminate all the isolated bright spot of the 100th line of the binarization image

(3)Scan the entire image to get the total number $\mathrm{C}$ of the luminescent spots of the 100th line

(4) Divide the value of the slope into 10 parts

(5) Allocate an accumulator for each of the $10 * \mathrm{C}$ space 
(6) Calculate slope for each of the $\mathrm{C}$ points with the other luminescent spots on the image and add 1 for the corresponding accumulator.

(7) Compare the values of the $10 * \mathrm{C}$ accumulators with one another to get the maximum number, and make a record of the corresponding point's column coordinate

(8) Take the above determined coordinates as the known fiducial mark, figure out the slope with all the bright spots, if the slope of the line for the points fell within the defined area, they are the bright spots, otherwise set the brightness of this value to zero.

Through a number of experimental verification, various methods of straight line detection are compared as following:

Table 2 Contrast of different algorithm

\begin{tabular}{lccc}
\hline & Classical HT & HT with kown point & Improved HT \\
\hline Processing time per frame & $3 \mathrm{~s}$ & $1.5 \mathrm{~ms}$ & $17.3 \mathrm{~ms}$ \\
Effect & best & good & fair \\
Suitable for real-time image processing & NO & NO & YES \\
\hline
\end{tabular}

Using the improved HOUGH transformation to get the straight line, Fig.4 shows the original and effect image.

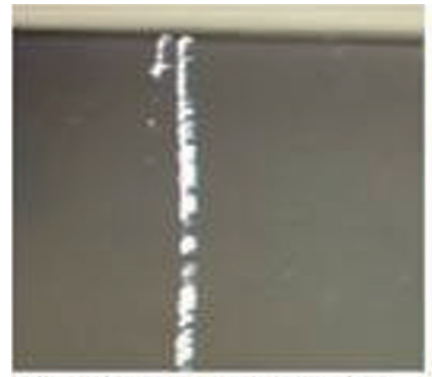

Result of binartion

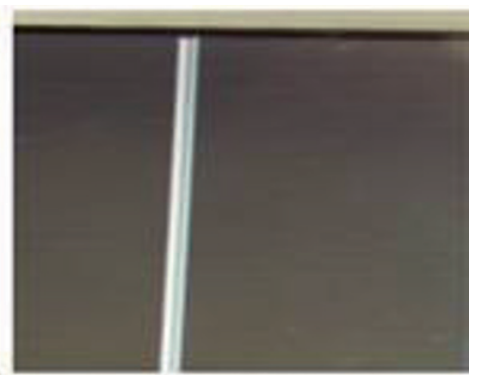

Result of improved HT

Fig. 4 Result of improved HT

\section{CONCLUSION}

In order to meet the demand for the Agriculture vehicle visual navigation, this paper provide a IDE based on dual core (DM642 DSP and ARM9) to solve the problem of image pretreatment and the extraction of navigation line in the processing of image encountered in agricultural visual navigation. By adopting Improved HOUGH transform, this algorithm not only reduce the storage space but also it can meet the demand of real-time. All of the 
work above make a good foundation for the ARM9 MCU to precisely locate the robot position and control the navigation. Meanwhile, the embedded system has good flexibility to facilitate the transplantation of farmland in the experimental environment. A large number of experiments proved that the method is effective and practical.

\section{ACKNOWLEDGEMENTS}

This work is supported by China high tech development plan "863"(NO:2006AA10A304).

\section{REFERENCES}

Gan-Mor S, R L,Clark. 2001 DGPS-based on automatic guidance-implementation and economical analysis, ASAE Paper, No.01-1192

Illingworth J, Kittlor J. A survey of the Hough transform, CVGIP,1988,44:87 116

Pinto F A C , Reid J F, Zhang Q. Vehicle guidance parameter determination from crop row images using principal component analysis, Journal of Agricultural Engineering Research,2000,75(3): 257 264

Toru Torii, Akirs Takamizawa. Crop row tracking by an autonomous vehicle using machine vision, JSAE,2000,62(5):37 42

Wilson J N. Guidance of agricultural vehicles-a historical perspective, Computers and Electronics in Agriculture,2000, 25 (1):3 $\sim 9$.

Zhang Wei, Du Shangfeng. Application of Hough transform in farmland machinery visual navigation, Instrumentation Journal, 2005,26(8): 706-707

Zhao Ying, Chen Bingqi, Wang Shumao, et al. Fast Detection of Furrows Based on Machine Vision on Autonomous Mobile Robot,Journal of Agricultural Machinery, 2006,37(4): $83 \sim 86$. 